\title{
DETERMINANTES DEL SOBREPESO Y LA OBESIDAD EN NIÑOS EN EDAD ESCOLAR EN PERÚ
}

\author{
DETERMINING FACTORS OF OVERWEIGHT AND OBESITY IN CHILDREN AT SCHOOL \\ AGE IN PERU
}

Monica L. Mispireta ${ }^{1, a}$

Division of Health Sciences, Idaho State University. Idaho, EE.UU.

Médico maestra en Ciencias de la Salud

Recibido: 21-06-12; Aprobado: 25-07-12

\begin{abstract}
RESUMEN
La obesidad en escolares es un problema que se encuentra en incremento en el Perú. Está concentrado en las zonas urbanas, principalmente en Lima donde uno de cada tres niños tiene exceso de peso. Un estudio inicial en 80 colegios de Lima mostró que más importante sería la falta de actividad física que el volumen de la ingesta para explicar el sobrepeso y la obesidad en escolares. Se requieren estudios más detallados al respecto. A pesar de la limitada información disponible de sus determinantes, es necesario implementar medidas culturalmente apropiadas para combatirla como parte de las políticas nutricionales actuales, y así prevenir que el problema continúe incrementándose y que la sostenibilidad del sistema de salud no se vea afectada.
\end{abstract}

Palabras clave: Obesidad; Sobrepeso; Niño; Estado nutricional; Actividad física (fuente: DeCS BIREME).

\begin{abstract}
Obesity in children at school age is an increasing problem in Peru. It concentrates in urban areas, mainly in Lima where one out of three children is overweight. An initial study in 80 schools in Lima showed that the lack of physical activity would have a greater impact on overweight and obesity in school children than the amount of food intake. More detailed studies are required. In spite of the limited information available regarding its determining factors, it is necessary to implement culturally-sensitive measures to fight this problem as part of the current nutritional policies, and prevent the problem from spreading, making sure the sustainability of the health system is not affected.
\end{abstract}

Key words: Obesity; Overweight; Child; Nutritional status; Motor activity (source: MeSH NLM).

\section{INTRODUCCIÓN}

No existe duda que la obesidad es un problema de salud pública a nivel global, y que se encuentra en incremento en muchos países en desarrollo. A nivel mundial, existen muchos estudios que han cuantificado el problema en la población adulta; sin embargo, para la población infantil, incluyendo escolares, la información es limitada (1). Además, en niños existe el problema de falta de concordancia en la definición de sobrepeso y obesidad, lo que hace problemático el estudio de tendencias y su comparación entre poblaciones ${ }^{(2)}$.

Dentro de la población infantil, un subgrupo con riesgo elevado de desarrollar obesidad son los niños en edad escolar. En los niños pequeños, el hogar es su principal ambiente físico y social. Con el paso de los años, el ambiente al que están expuestos se va ampliando y se vuelve más complejo y menos controlado. Las percepciones y decisiones nutricionales, así como de actividad física son afectadas por el ambiente y en los niños en edad escolar están determinadas principalmente por las opciones disponibles en el hogar, la comunidad y la escuela ${ }^{(3)}$. La complejidad de la interrelación de estos factores resulta desafiante al momento de desarrollar e implementar programas enfocados a tratar y prevenir la obesidad en este grupo poblacional.

En Perú, la mayoría de información nutricional recolectada de manera sistemática proviene de las encuestas demográficas y de salud familiar (ENDES) y de las encuestas del Monitoreo Nacional de Indicadores Nutricionales (MONIN) ${ }^{(4)}$, que se concentran en los mismos grupos poblacionales que las ENDES. La

Citar como: Mispireta ML. Determinantes del sobrepeso y la obesidad en niños en edad escolar en Perú. Rev Peru Med Exp Salud Publica. 2012;29(3):361-5. 
cantidad de información disponible sobre la prevalencia y determinantes de obesidad en niños en edad escolar en Perú es muy limitada; por lo tanto, no se conoce con exactitud la extensión del problema, ni las tendencias y factores asociados a este. Entre los años 1998 y 2001, como parte del MONIN, se recolectó información nutricional de niños en edad escolar (de cinco a catorce años de edad); de quienes residían con menores de cinco años, y de mujeres en edad fértil (5). Los niños fueron clasificados con riesgo de sobrepeso si se encontraban en el percentil 85-94 del Índice de Masa Corporal (IMC), y con sobrepeso si se hallaban en el percentil 95 o más, usando como referencia las curvas de crecimiento recomendadas por la Organización Mundial de la Salud (OMS) desarrolladas por el Centro Nacional de Estadísticas de Salud de los Estados Unidos (National Center for Health Statistics $\mathrm{NCHS})^{\left({ }^{6}\right)}$. A nivel nacional, se encontró un incremento de $0,7 \%$ en la prevalencia de riesgo de sobrepeso entre $1998(9,9 \%)$ y $2001(10,6 \%)$, y un incremento de $1,2 \%$ en la prevalencia de sobrepeso (3,2\% en 1998 y $4,4 \%$ en 2001). Al evaluar tendencias de acuerdo a región, no se encontró un incremento significativo en la prevalencia de riesgo de sobrepeso o sobrepeso en la mayoría de regiones; sin embargo, la prevalencia de sobrepeso en Lima Metropolitana se incrementó a más del doble en el periodo de estudio (de 5,6\% en 1998 a 12,2\% en 2001). A pesar de que el proceso de selección no produce una muestra representativa de la población en edad escolar de Perú, debido a que la metodología de selección fue la misma para los MONIN 1998-2001, y la definición de riesgo de sobrepeso y sobrepeso son consistentes en sus informes, resulta adecuado hacer un análisis de tendencias usando estos datos. Lamentablemente, los datos no fueron presentados de acuerdo al área de residencia (urbano/rural), o al sexo.

En 2007, se realizó un estudio de prevalencia de sobrepeso y obesidad representativo de escolares entre $3 .^{\text {er }}$ a $6 .^{\circ}$ grados de primaria (7-14 años de edad) en Lima Metropolitana y Callao ${ }^{(7)}$. En este estudio también se clasificó sobrepeso y obesidad empleando como referencia las curvas de crecimiento recomendadas por la OMS ${ }^{(6)}$, y se encontró una prevalencia de 20,6\% de sobrepeso y $15,5 \%$ de obesidad. La prevalencia de sobrepeso y obesidad fue mayor en las escuelas privadas que en las estatales (obesidad: $23,3 \%$ frente a $18,3 \%$, sobrepeso: $21,6 \%$ frente a $10,3 \%$ ), no hubo diferencias en la prevalencia de sobrepeso de acuerdo a sexo, aunque sí para obesidad, pues fue mayor en hombres ( $19 \%$ en hombres y $12 \%$ en mujeres). Los datos más recientes de prevalencia de obesidad en niños en edad escolar en Perú corresponden a los de la Encuesta

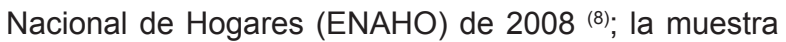
incluida es representativa de los hogares de Perú. En este estudio se definió sobrepeso usando también como referencia las curvas de crecimiento recomendadas por la OMS; sin embargo, no se menciona el punto de corte usado para definir sobrepeso; en el grupo de niños de 5-9 años de edad se encontró una prevalencia de sobrepeso de $7,8 \%$ a nivel nacional, la cual fue mayor en los residentes de área urbana $(11,4 \%)$ que en los de área rural $(2,7 \%)$. Adicionalmente se encontró que la prevalencia de sobrepeso disminuye con decrecientes niveles de pobreza extrema $(2,1 \%$ en pobres extremos, $5,4 \%$ en pobres no extremos, y $11,8 \%$ en no pobres).

A pesar de la escasa información disponible de obesidad en los niños en edad escolar, y la falta de consistencia en su definición, existe evidencia razonable para concluir que obesidad en escolares es un problema que se encuentra en incremento en el Perú, y que está concentrado en las zonas urbanas, principalmente en Lima. Estos resultados son consistentes con la información disponible en niños menores de cinco años y mujeres en edad fértil, los cuales también muestran mayores incrementos en la prevalencia de sobrepeso y obesidad concentrados en zonas urbanas, principalmente en Lima ${ }^{(9)}$. Dado que Lima contiene aproximadamente la cuarta parte de la población de Perú, resulta necesario cuantificar mejor la extensión del problema en este grupo poblacional y mejorar nuestro nivel de entendimiento de sus determinantes para implementar medidas culturalmente apropiadas dirigidas a reducir y prevenir el problema de la obesidad infantil.

\section{DETERMINANTES DE OBESIDAD EN NIÑOS EN EDAD ESCOLAR EN PERÚ}

Perú, al igual que muchos países en desarrollo, está sobrellevando cambios en los patrones de alimentación y actividad física que se ven asociados con un incremento en sobrepeso y obesidad en la población ${ }^{(9)}$. Si bien un balance calórico positivo es el principal determinante de la ganancia excesiva de peso, existen factores socioeconómicos, ambientales y culturales que también son importantes para el desarrollo de sobrepeso y obesidad.

Al igual que los datos de prevalencia y tendencias de obesidad en niños en edad escolar en Perú, la información disponible de sus determinantes es limitada. En el mismo estudio realizado en escolares de $3 .^{\text {er }}$ a $6 .^{\circ}$ de primaria en 2007 , se comparó ingesta y actividad física en una submuestra aleatoria de niños con obesidad (percentil IMC > 95) y otra, con peso adecuado (IMC entre percentil 18 y 85) ${ }^{(7)}$. En este estudio se encontró que en promedio los niños con obesidad ingerían 200 kilocalorías (kcal) menos $(p=0,03)$ y gastaban $225 \mathrm{kcal}$ más de actividad física $(p=0,02)$ por día, al compararlos con los niños de 
peso adecuado. Sin embargo, al tomar en cuenta el peso del niño, los niños de peso adecuado gastaron en promedio $67 \%$ más calorías de actividad física por kilo de peso que los niños con obesidad. Dato importante fue que independientemente del peso del niño, menos del $1 \%$ de los niños cumplió con las recomendaciones de actividad física para su edad. En promedio, las niñas realizaron menos actividad física que los niños, y los niños que acuden a escuelas privadas realizaron menos actividad física que los que acuden a escuelas estatales.

Si bien estos resultados dan información importante sobre la falta de actividad física como posible determinante del problema de obesidad en escolares peruanos, resulta crítico estudiarlo en mayor detalle usando una perspectiva sociocultural. Entre otros, debería explorarse las oportunidades para realizar actividad física en la escuela, como parte del receso o del curso de Educación Física. En general, en las escuelas estatales es común la falta de espacios recreacionales, así como la carencia de artículos deportivos para realizar actividades estructuradas. Los alimentos en dichas escuelas provienen de programas nacionales como Desayunos Escolares o son adquiridos en la cafetería escolar, que en general no contienen vegetales o frutas. También, es importante estudiar el contenido de los alimentos consumidos por los niños en bodegas o vendedores ambulantes en su camino de la escuela al hogar. Con frecuencia estos alimentos son altamente calóricos y pobremente nutricionales por consistir principalmente en productos basados en hidratos de carbono azucarados. Por último, es importante entender en mayor detalle la percepción que tienen los niños de su peso corporal y la percepción que tienen sus padres del mismo; y su asociación con bienestar, salud y enfermedad. Asociaciones de sobrepeso con salud, o falta de preocupación de los padres acerca del exceso de peso en sus hijos, se han identificado como potenciales barreras al momento de implementar programas nutricionales enfocados en prevenir obesidad en niños ${ }^{(10)}$.

\section{LECCIONES APRENDIDAS DE INTERVENCIONES PARA COMBATIR LA OBESIDAD INFANTIL EN ESCOLARES EN LOS PAÍSES DESARROLLADOS}

A pesar de la escasa información (y sus limitaciones metodológicas), sobre la obesidad en escolares en Perú, resulta claro que es un problema que se encuentra en incremento mayormente en las zonas urbanas, y principalmente en Lima. La falta de programas específicos para combatir este problema nos indica que no está recibiendo la atención requerida. La obesidad está asociada con un alto costo económico y de capital humano por su impacto psicosocial así como en morbilidad y mortalidad. Resulta imperativo que se incluyan medidas para prevenir y tratar la obesidad infantil como parte de las políticas nutricionales actuales para evitar que el problema continúe incrementándose y que la sostenibilidad del sistema de salud se vea afectada.

Numerosas intervenciones han sido implementadas en diferentes niveles, incluyendo múltiples componentes, principalmente en países desarrollados (11). Si bien suponemos que los determinantes de obesidad en países desarrollados son diferentes a los de los países en desarrollo, debemos aprender y tomar en cuenta las intervenciones que han resultado exitosas en otros contextos al momento de diseñar e implementar las nuestras. La mayoría de programas para combatir la obesidad en niños en edad escolar se encuentra a nivel de escuela (11). Las escuelas resultan un lugar intuitivo para la implementación de las intervenciones porque pueden llegar a un grupo poblacional grande en un ambiente controlado, son idóneas para incluir múltiples componentes incluyendo actividad física y alimentación y, sobre todo, porque tienen potencial para ser sostenibles a largo plazo si logran institucionalizarse formalmente. La mayoría de los programas escolares tienen entre sus componentes a la educación en nutrición y el incremento en la actividad física. Con menor frecuencia se incluye modificación de la infraestructura como parte de la intervención. La gran mayoría de intervenciones en la escuela han mostrado una mejora modesta en composición corporal y aptitud física, principalmente en las que lograron un incremento en la actividad física en los niños ${ }^{(12-14)}$. Adicionalmente, la mayoría de estas intervenciones han tenido un impacto positivo en el comportamiento y educación nutricional. La mayoría de las intervenciones disponibles en la literatura tienen un período de seguimiento corto (menor de un año) por lo que no se conoce con exactitud su impacto y sostenibilidad a largo plazo. Sin embargo, los resultados positivos en su implementación, su proceso e impacto, son alentadores, por ello, se debe continuar con la intervención para generar evidencia de su efectividad a largo plazo. Más aun, los pocos estudios con período de seguimiento prolongado han demostrado mejora en composición corporal a corto y largo plazo; pero, de mayor importancia, fue el impacto positivo en presión arterial y lípidos séricos, más allá de tres años de implementado el programa ${ }^{(15-17)}$.

Las intervenciones para combatir la obesidad en escolares, en el hogar y la comunidad, son limitadas. Sin embargo, existen programas escolares con componentes 
adicionales para el hogar o la comunidad. La evidencia sugiere que involucrar a la familia como parte de las intervenciones en la escuela puede tener un impacto positivo. Específicamente, se ha encontrado que incluir a la familia en diferentes actividades implementadas en la escuela logra reducir sustancialmente el tiempo de inactividad e incrementar la actividad física de los niños ${ }^{(14,18)}$. Existe un número limitado de intervenciones en la escuela con un componente importante de comunidad. En general, se ha obtenido un impacto modesto en composición corporal ${ }^{(19)}$. Intervenciones aisladas en la comunidad, enfocadas en incrementar opciones saludables de alimentos en bodegas, o mejorar la infraestructura disponible en la comunidad para realizar actividad física, no han tenido resultados muy alentadores (20); sin embargo, los resultados podrían mejorar si las intervenciones en la comunidad se desarrollan con participación de la población general en su implementación, o si se asocian a intervenciones escolares (21). Sin duda, este tipo de intervenciones necesitan una mayor exploración y constituyen una importante área de expansión dentro de las actividades para combatir la obesidad infantil.

\section{CONCLUSIONES}

La obesidad de niños en edad escolar en Perú es un problema creciente que tiene mayor prevalencia en las zonas urbanas, sobre todo en Lima. No existe solución simple y única para disminuir la prevalencia de obesidad infantil. Sin embargo, consideramos que existen cambios pequeños y de bajo costo que se podrían implementar de manera relativamente rápida. En las escuelas es necesario reestructurar el currículo del curso de Educación Física para incrementar la calidad y duración de actividad física dentro de los periodos ya asignados. A su vez, es necesario promover actividades que impliquen incremento en la actividad física durante el receso o como parte de actividades extracurriculares, además de acondicionar las escuelas con áreas más adecuadas y artículos deportivos para incrementar la actividad física en los niños. Adicionalmente, es necesario educar a los niños y sus padres con el fin de mejorar su conocimiento nutricional, haciendo énfasis en lograr una combinación balanceada en los alimentos ofrecidos en el hogar y en las loncheras. También se debe incrementar las opciones saludables en los alimentos ofrecidos en los quioscos escolares, así como a través de los desayunos escolares; adicionalmente, mejorar la ubicación de los alimentos en los puntos de venta dentro de la escuela con el fin de hacer más visibles las opciones saludables. Si bien estas actividades deberían ser implementadas en las escuelas, con apoyo del Estado, es necesario involucrar a la sociedad civil para lograr su aceptación e integración en la comunidad.

Fuentes de financiamiento: autofinanciado.

Conflictos de interés: el autor declara no tener ningún conflicto de interés.

\section{REFERENCIAS BIBLIOGRÁFICAS}

1. World Health Organization. Obesity: preventing and managing the global epidemic. Report of a WHO consultation. Geneva: World Health Organization; 2000.

2. Flegal KM, Ogden CL. Childhood obesity: are we all speaking the same language? Adv Nutr. 2011;2(2):159S$66 S$.

3. Birch LL, Davison KK. Family environmental factors influencing the developing behavioral controls of food intake and childhood overweight. Pediatr Clin North Am. 2001;48(4):893-907.

4. Instituto Nacional de Salud (INS). Monitoreo Nacional de Indicadores Nutricionales (MONIN) [Internet]. Lima: Instituto Nacional de Salud; [citado el 14 de junio de 2012].
Disponible en: http://www.ins.gob.pe/ portal/jerarquia/5/311/monitoreonacional-de-indicadores-nutricionalesmonin/jer.311

5. Instituto Nacional de Salud (INS), Centro Nacional de Alimentación y Nutrición. Monitoreo Nacional de Indicadores Nutricionales, Perú $1997-$ 2001. Lima: INS; 2006.

6. de Onis M, Onyango AW, Borghi E, Siyam A, Nishida C, Siekmann J. Development of a WHO growth reference for school-aged children and adolescents. Bull World Health Organ. 2007;85(9):660-7.

7. Liria MR, Mispireta ML, Lanata CF, Creed-Kanashiro HM. Perfil Nutricional en escolares de Lima y Callao. Lima: Instituto de Investigación Nutricional; 2008.
8. Instituto Nacional de Salud (INS), Centro Nacional de Alimentación y Salud. Informe final: Perfil nutricional y pobreza en Perú. Lima: INS; 2009.

9. Mispireta M, Rosas A, Velasquez J, Lanata C. Transición nutricional en el Perú, 1991 - 2005. Rev Peru Med Exp Salud Publica. 2007;24(2):129-35.

10. Towns N, D'Auria J. Pareantal perceptions of their child's overweight: an integrative review of the literature. J Pediatr Nurs. 2009; 24(2):115-30.

11. Flynn MA, McNeil DA, Maloff B, Mutasingwa D, Wu M, Ford C, et al. Reducing obesity and related chronic disease risk in children and youth: a synthesis of evidence with 'best practice' recommendations. Obes Rev. 2006;7 Suppl 1:7-66. 
12. Arbeit ML, Johnson CC, Mott DS, Harsha DW, Nicklas TA, Webber LS, et al. The Heart Smart cardiovascular school health promotion: behavior correlates of risk factor change. Prev Med. 1992;21(1):18-32

13. Dwyer T, Coonan WE, Leitch DR, Hetzel BS, Baghurst RA. An investigation of the effects of daily physical activity on the health of primary school students in South Australia. Int J Epidemiol. 1983;12(3):308-13.

14. Manios Y, Moschandreas J, Hatzis C, Kafatos A. Evaluation of a health and nutrition education program in primary school children of Crete over a three-year period. Prev Med. 1999;28(2):149-59.

15. Alexandrov AA, Maslennikova GY, Kulikov SM, Propirnij GA, Perova NV. Primary prevention of cardiovascular disease: 3-year intervention results in boys of 12 years of age. Prev Med. 1992;21(1):53-62.

16. Bush PJ, Zuckerman AE, Taggart VS, Theiss PK, Peleg EO, Smith SA. Cardiovascular risk factor prevention in black school children: the "Know Your Body" evaluation project. Health Educ Q. 1989;16(2):215-27.

17. Walter HJ, Hofman A, Vaughan RD, Wynder EL. Modification of risk factors for coronary heart disease. Five-year results of a school-based intervention trial. $\mathrm{N}$ Engl J Med. 1988;318(17):1093-100.

18. Golan M, Crow S. Targeting parents exclusively in the treatment of childhood obesity: long-term results. Obes Res. 2004;12(2):357-61.

19. Gittelsohn J, Park S. School- and community-based interventions. In:
Freemark M, editor. Pediatric obesity: etiology, pathogenesis, and treatment. 1 st ed. New York: Springer; 2010. p. 315-35.

20. Gittelsohn J, Rowan M, Gadhoke P. Interventions in small food stores to change the food environment, improve diet, and reduce risk of chronic disease. Prev Chronic Dis. 2012;9:E59.

21. Gittelsohn J, Davis SM, Steckler A, Ethelbah B, Clay T, Metcalfe L, et al. Pathways: lessons learned and future directions for school-based interventions among American Indians. Prev Med. 2003;37(6 Pt 2):S107-12.

Correspondencia: Monica L Mispireta Dirección: 921 South 8th Avenue, Stop 8002, Pocatello, Idaho 83209-8002, EE. UU.

Teléfono: (208) 2825601

Correoelectrónico:mispmoni@isu.edu

\section{Visite nuestra página en Facebook, www.facebook.com/rpmesp Infórmese sobre los eventos y los nuevos contenidos de la Revista Peruana de Medicina Experimental y Salud Pública}

\section{REVISTA PERUANA DE MEDICINA EXPERIMENTAL Y SALUD PÚBLICA} Difundiendo investigación en salud
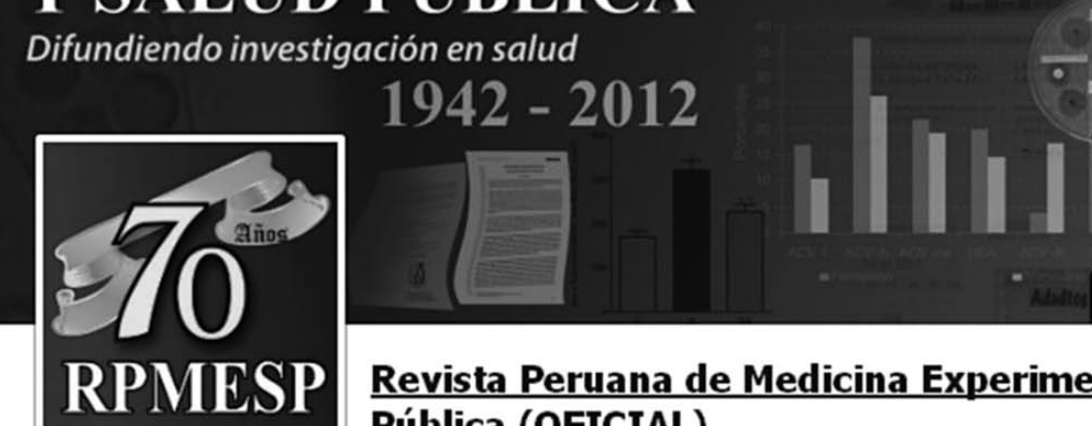

\section{Revista Peruana de Medicina Experimental y Salud Pública (OFICIAL)}

A 325 personas les gusta esta página $\cdot 22$ personas están hablando sobre esto

Comunidad [?]

La Revista Peruana de Medicina Experimental y Salud Publica es el órgano oficial de difusión científica del Instituto

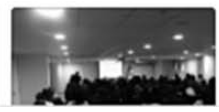

